\title{
Metabolic Profiling of the Rat Liver After Chronic Ingestion of Alpha-Naphthylisothiocyanate Using In Vivo and Ex Vivo Magnetic Resonance Spectroscopy
}

\author{
Bhavana S. Solanky ${ }^{\star}, 1$, Gina J. Sanchez-Canon ${ }^{\star}$, Jeremy F. L. Cobbold ${ }^{\dagger}$, Simon D. Taylor- \\ Robinson $^{\dagger}$, Jimmy D. Bell ${ }^{\ddagger}$, Cheryl L. Scudamore ${ }^{\S}$, Eleanor Ross $₫$, Julie C. Holder $\rrbracket$, Po- \\ Wah So ${ }^{*}, \|$, and I. Jane Cox ${ }^{\|} \|$ \\ *Imaging Sciences Department, Institute of Clinical Sciences, Imperial College London, \\ Hammersmith Hospital Campus, London W12 0NN, U.K. \\ tLiver Unit, Division of Diabetes Endocrinology and Metabolism, Department of Medicine, \\ Imperial College London, St Mary's Hospital Campus, London W2 1PG, U.K. \\ $\ddagger$ Metabolic and Molecular Imaging Group, MRC Clinical Sciences Centre, Imperial College \\ London, London W12 ONN, U.K. \\ §Pathology and Infectious Diseases, Royal Veterinary College, Hatfield, Hertfordshire AL9 7TA, \\ U.K. \\ ISafety Assessment, GlaxoSmithKline Pharmaceuticals, Stevenage, Hertfordshire SG12 0DP, \\ U.K. \\ "Department of Neuroimaging, Institute of Psychiatry, King's College London, Denmark Hill \\ Campus, London SE5 9NU, U.K. \\ ${ }^{I I}$ Foundation for Liver Research, Institute of Hepatology, London WC1E 6HX, U.K.
}

\section{Abstract}

Certain human diseases affecting the biliary tree can be modeled in rats by ingestion of the hepatobiliary toxin alpha-naphthylisothiocyanate (ANIT). Phosphorus magnetic resonance spectroscopy (MRS) allows the noninvasive monitoring of cell dynamics through detection of phosphodiesters (PDE) and phosphomonoesters (PME). Hepatic ${ }^{31} \mathrm{P}$ MRS techniques were therefore used to study the toxic effects of low-dose chronic ANIT ingestion, with a view toward providing biomarkers sensitive to hepatobiliary dysfunction and cholestatic liver injury. Rats were fed an ANIT supplemented diet at three doses (ANIT_0.05\%, ANIT_0.04\%, and ANIT_0.025\%) for 2 weeks. Data from in vivo MRS were compared with results from pair-fed controls (PFCs). Blood and tissue samples were collected at 2 weeks for clinical chemistry, histology, and ${ }^{1} \mathrm{H}$ magic angle spinning MRS. Increases in PDE, relative to total phosphorus ( $\mathrm{tPh})$, were detected in both the ANIT_0.05\% and ANIT_0.04\% groups $(0.07 \pm 0.01$ and $0.08 \pm 0.01$, respectively $)$ relative to PFC groups $(0.03 \pm 0.01$ and $0.05 \pm 0.01$, respectively). An increase in PME/tPh was observed in the ANIT_ $0.05 \%$ group only $(0.17 \pm 0.02)$ relative to PFC_0.05\% $(0.12 \pm 0.01)$. Ex vivo ${ }^{1} \mathrm{H}$ MRS findings supported this, wherein measured phosphocholines (PCs) were increased in ANIT_0.05\% and ANIT_0.04\% groups. Increases in relative total choline (tCho) distinguished the ANIT_ $0.05 \%$ group from the ANIT_0.04\% group. Markers of hepatotoxicity such as raised total bilirubin and alkaline phosphatase were found at all ANIT doses. Histological findings included a

\footnotetext{
(C) The Author 2012. Published by Oxford University Press on behalf of the Society of Toxicology. All rights reserved.

${ }^{1}$ To whom correspondence should be addressed at NMR Research Unit, Department of Neuroinflammation, Institute of Neurology, University College London, Queen Square, London WC1N 3BG, U.K. Fax: +0207-278-5069. b.solanky@ucl.ac.uk. .
} 
dose-related increase in both severity of biliary hyperplasia and focal hepatocellular necrosis. Here, we found that ANIT-induced moderate hepatobiliary dysfunction was associated with a relative increase in phosphodiesters in vivo and PCs ex vivo. Raised PME/tPh in vivo and tCho ex vivo were also present at high doses corresponding to a higher incidence of marked biliary hyperplasia and moderate hepatocellular necrosis.

\section{Keywords}

alpha-naphthylisothiocyanate; hepatobiliary; hyperplasia; magnetic resonance spectroscopy; phosphorus; rats

Hepatobiliary injury is commonly encountered in response to certain drugs and toxins (Plaa and Priestly, 1976) frequently producing a syndrome of intrahepatic cholestasis in humans and, histologically, with biliary hyperplasia in animal models. Thus, it is crucial to screen for such adverse events early during preclinical drug development to increase efficiency and ultimately reduce failure in subsequent clinical studies. The ability to detect biliary hyperplasia and associated hepatobiliary injury noninvasively, by longitudinal liver-specific assessment, would be of value in the development of novel therapies and aid toward the understanding of hepatic pathophysiological processes.

Alpha-naphthylisothiocyanate (ANIT) has been studied extensively as a model of chemical cholestasis in susceptible species, such as the rat (Becker and Plaa, 1965; Dahm et al., 1991; Indacoch and Plaa, 1971; Krell et al., 1982). Bile duct obstruction has been observed to result from the sloughing of necrotic biliary epithelial cells into the duct lumen following ANIT treatment (Lopez and Mazzanti, 1955). In the acute setting, it causes mast cell infiltration and accumulation of epithelial debris leading to cholestasis, whereas chronic ingestion leads to ductal proliferation, hepatocellular necrosis, and periductal inflammation (Goldfarb et al., 1962).

Noninvasive measures have been sought to assess ANIT-induced hepatotoxicity, and magnetic resonance spectroscopy (MRS) techniques have the potential to provide such biomarkers. For example, markers for cell synthesis and degradation in the magnetic resonance spectrum of liver are well documented (Bell et al., 1993; Waters et al., 2001, 2002). Previous studies have used MRS to study liver extracts and intact liver tissue, as well as urine and serum, for up to 7 days after a single chronic dose of ANIT (typically 150-200 $\mathrm{mg} / \mathrm{kg}$ ) (Beckwith-Hall et al., 1998; Waters et al., 2001, 2002). Such studies in liver tissue have shown initial lipid increases followed by a decrease to below control levels by 7 days, suggesting hepatic steatosis associated with hyperlipidemia, an immediate reduction in glucose/glycogen (Glc/Glyc) concentration before recovery by 7 days, and persisting elevated phosphocholine (PC)/choline, trimethylamine-N-oxide, and betaine (Waters et al., 2001, 2002). MRS studies of urine have shown time-dependent changes from control in glucose, bile acids, citrate, 2-oxoglutarate, creatine, succinate, acetate, and hippurate (Beckwith-Hall et al., 1998; Waters et al., 2001). Similar biofluid changes have been observed with other model hepatotoxins including galactosamine (Sequeira et al., 1990). A few studies have been performed at lower single doses of ANIT, and effects on urinary metabolites were observed in the absence of changes evident by clinical chemistry or microscopic analysis after a single ANIT dose of $10 \mathrm{mg} / \mathrm{kg}$ (Robertson et al., 2000).

Cholestasis has been characterized following low-dose oral administration of ANIT (1 g/kg of powdered rat chow, equivalent to $0.1 \%$ ANIT) in a rat model by 7 and 14 days, showing cholestasis resulting in increased total bilirubin (TBil) and alanine transaminase (ALT), damaged bile ducts and significant bile duct proliferation (Tjandra et al., 2000). The 
associated metabolic changes, measured by MRS of the liver, for example, have not been previously documented in such a low-dose ANIT model to our knowledge.

As ex vivo MRS studies of liver tissue have been found to be useful in interpreting hepatotoxic damage, there is scope for translation of such findings to in vivo hepatic MR study. Clinical hepatic phosphorus-31 MRS ( ${ }^{31} \mathrm{P}$ MRS) enables the assessment of a number of intermediates involved in hepatic cellular metabolism, such as phosphomonoesters (PME) and phosphodiesters (PDE) (Angus et al., 1990; Changani et al., 1998; Corbin et al., 2003; Jalan et al., 1996a; Munakata et al., 1993). Its utility in the assessment of a number of chronic liver diseases has been demonstrated (Angus et al., 1990; Jalan et al., 1995, 1996b,c; Menon et al., 1995; Taylor-Robinson et al., 1997). Importantly, the PDE resonance contains information from cell membrane breakdown products and biliary phospholipids. In cholestatic conditions, such as cholestatic liver transplant patients with chronic graft rejection, an increase in \% PDE was observed rather than a decrease as in cirrhosis (TaylorRobinson et al., 1998).

On the basis that in vivo ${ }^{31} \mathrm{P}$ MRS allows serial measurements and can be liver specific, we hypothesized that hepatic ${ }^{31} \mathrm{P}$ MRS would provide a useful biomarker for the serial assessment of cholestatic liver injury in the rat induced by low-dose chronic ANIT ingestion. Hence, the aims of this study were to investigate the use of in vivo hepatic ${ }^{31} \mathrm{P}$ MRS to provide potential biomarkers for hepatobiliary injury linked to biliary hyperplasia in the ANIT-fed rat model and to investigate longitudinal changes according to dose over a 2week time period. The hepatic ${ }^{31} \mathrm{P}$ MR findings were compared with ex vivo ${ }^{1} \mathrm{H}$ MR findings of intact liver tissue, using magic angle spinning (MAS) MR techniques, to provide both complementary and confirmatory information. Routine clinical chemistry and histopathology provided an assessment of the extent of ANIT-induced hepatobiliary damage.

\section{MATERIALS AND METHODS}

\section{Animals and treatment}

All experiments were performed in compliance with the U.K. Animals Scientific Procedures Act 1986. The doses in these studies were limited as higher doses of ANIT caused a decrease in body weight, which was greater than the limit of weight loss allowed by the Home Office animal license.

Sixty male Sprague Dawley rats (150-180 g; Charles River U.K. Ltd) were divided into six equal groups of $n=10$ per group. Adolescent rats were used as they offered a superior filling factor in the coil used for MRS and less spectral contamination from the tissue between the coil and the liver and were in keeping with animal weights in ANIT experiments by Goldfarb et al. (1962). Animals were paired and housed in standard open cages with a 12-h light/12-h dark cycle. All rats were initially fed ad libitum on a normal control diet (2014, 14\% Teklad protein rodent maintenance diet; Harlan Laboratories, U.K.). Cages were lined with white paper in order to easily identify and weigh spillages of food. The lining paper was changed daily in addition to changes due to wetness. Animals were provided with water ad libitum during the acclimatization period of 7 days and throughout the study.

Three groups ( $n=10$ per group) were then fed a diet supplemented with ANIT at 0.05, 0.04, and $0.025 \%$ of ANIT per kilogram of normal control diet (ANIT_0.05\%,ANIT_0.04\%, and ANIT_0.025\%, respectively) for 14 days. Body weight and food intake were recorded daily, and the average weekly intake was found (results for animals with no in vivo MRS data were excluded). As ANIT is known to suppress appetite, three pair-fed control (PFC) groups 
were also set up for each ANIT-dosed group, to control for reduced food intake by ANIT dosing (Leonard et al., 1981). PFC_0.05\%, PFC_0.04\%, and PFC_0.025\% animals were fed the non-ANIT supplemented (normal) diet, but the amount of food given was the same as that for the corresponding ANIT group and was adjusted daily.

\section{In vivo hepatic ${ }^{31} \mathrm{P}$ MRS}

Prior to in vivo ${ }^{31} \mathrm{P}$ MRS study, the animals were fasted for $15 \mathrm{~h}$ and then anesthetized with a $21 / \mathrm{min}$ oxygen and 4-5\% isoflurane mix. Anesthesia was maintained with a $1 \mathrm{l} / \mathrm{min}$ oxygen and 2-2.5\% isoflurane mix via a nose cone. Body temperature was controlled and respiration monitored. A customized dual surface coil, tuned to ${ }^{31} \mathrm{P}$ and ${ }^{1} \mathrm{H}$ frequencies (3 cm diameter [Magnetic Resonance Labs, Oxford, U.K.]), was used on a horizontal 4.7T Varian Inova System (Varian Inc., Palo Alto, CA) with a 20-cm bore size. The surface coil was curved to achieve a better filling factor and was placed underneath the rat torso for localization of the hepatic region. The field was shimmed to $<100 \mathrm{~Hz}$, measured by the width at half height of the water peak. Pilot MR images were collected for correct positioning of the coil over the liver, using a multislice gradient echo sequence with repetition time $(\mathrm{TR})=173 \mathrm{~ms}$, echo time $(\mathrm{TE})=4.89 \mathrm{~ms}$, a flip angle of $30^{\circ}, 30 \times 30 \mathrm{~mm}$ field of view, $128 \times 128$ matrix size, 20 consecutive transverse slices, and 6 averages. Following identification of appropriate coil placement, unlocalized hepatic ${ }^{31} \mathrm{P}$ MR spectra were then acquired using a simple pulse-acquire sequence, with a pulse optimized to give a $90^{\circ}$ excitation in the liver region with the added advantage of overtipping signal from the abdominal region. Respiratory gating was not used as the spectra acquired were unlocalized and hence the resulting spectrum was the average ${ }^{31} \mathrm{P}$ MR signal from the sensitive area of the coil, which was optimized to have the largest signal $\left(90^{\circ}\right.$ pulse) in the liver. Given that the liver of each animal is large compared with the field of view of the coil, it is fair to assume that the region optimized to have a $90^{\circ}$ excitation will remain within the liver throughout scanning even on different phases of the respiration cycle. Had respiratory gating been included scan time will have increased. ${ }^{31} \mathrm{P}$ MRS data were collected with a TR of $5 \mathrm{~s}$, 544 averages, and a spectral width of $6000 \mathrm{~Hz} .{ }^{31} \mathrm{P}$ MRS was performed at baseline and weeks 1 and 2 after the start of the supplemented diet.

Data were analyzed in the time domain using a Java-based graphical user interface jMRUI analysis package and the AMARES algorithm, after conversion of the data in MatLab to allow software compatibility (Naressi et al., 2001; Vanhamme et al., 1997). All resonances were referenced using phosphocreatine (PCr) at $0 \mathrm{ppm}$. The $\mathrm{PME}$, inorganic phosphate $(\mathrm{Pi})$, $\mathrm{PDE}, \mathrm{PCr}$, and the three nucleotide triphosphate (mainly adenosine triphosphate) peaks in the spectrum were fitted after application of 5- $\mathrm{Hz}$ line broadening and manual phasing. The total liver ${ }^{31} \mathrm{P}$ signal, $\mathrm{tPh}$, was obtained by summation of the peak integrals and subtraction of the nonhepatic PCr component, and the ratios of PME/tPh and PDE/tPh were calculated. PCr was checked to be constant so it could be fair to assume the contribution of abdominal signal was constant for all other peaks between groups.

\section{Serum and liver tissue collection}

After the final MRS scan at 2 weeks, animals were sacrificed by ip injection of pentobarbital $(0.5 \mathrm{ml} / 300 \mathrm{~g})$ under isoflurane anesthesia. Approximately $3 \mathrm{ml}$ of blood was collected by cardiac puncture and/or the vena cava and allowed to clot overnight $\left(4^{\circ} \mathrm{C}\right)$ before centrifugation to obtain serum. Samples were then frozen immediately at $-80^{\circ} \mathrm{C}$ to avoid degradation prior to clinical chemistry, which was conducted off site.

The liver was harvested, and three samples from the median lobe were excised. These were snap frozen in liquid nitrogen and stored at $-80^{\circ} \mathrm{C}$ for subsequent MAS MRS or fixed in $10 \%$ neutral-buffered formalin for histopathology. 


\section{Serum analysis}

Total bilirubin (TBil), albumin (ALB), alanine transaminase (ALT), aspartate transaminase (AST), alkaline phosphatase (ALP), and total cholesterol (Chol) levels were measured using routine clinical chemistry methodologies (Balazs et al., 1961).

\section{Histopathology}

Formalin-fixed liver samples were embedded into paraffin wax prior to sectioning into $5 \mu \mathrm{m}$ slices. The sections were stained with hematoxylin and eosin prior to histological assessment by light microscopy. The sections were assessed by a single observer (E.R.) and peer reviewed by an experienced pathologist (C.L.S.). Pathological changes were scored out of a maximum of 5 where $\mathrm{NAD}=$ no abnormalities detected, $1=$ minimal, 2 mild, $3=$ moderate, and $4=$ marked change present.

\section{Ex vivo hepatic ${ }^{1} \mathrm{H}$ MAS MRS of liver tissue}

EX vivo ${ }^{1} \mathrm{H}$ MAS MRS was chosen over in vivo ${ }^{1} \mathrm{H}$ MRS due to its higher spectral resolution permitting an accurate and more comprehensive account of the ${ }^{1} \mathrm{H}$ metabolic profile, particularly choline-containing resonances and lipid moieties. The ex vivo ${ }^{1} \mathrm{H}$ MAS MR spectra improve understanding of the ANIT-induced metabolic changes and are an advanced means to corroborate and aid interpretation of the in vivo ${ }^{31} \mathrm{P}$ data.

Liver samples $(15-19 \mathrm{mg})$ were randomly selected from each experimental $(n=6)$ and PFC group $(n=6)$ for MAS MRS analysis. All samples, except one, were from animals included in the in vivo MRS results, and all matched the samples used for histology. MAS MRS data were acquired on a vertical 11.7T (51 mm bore) JEOL ECP system (Tokyo, Japan) fitted with an MAS DOTY probe (Columbia, TN). The liver samples were thawed and then placed in a $20-\mu \mathrm{l}$ sealing cell within a $4 \mathrm{~mm}$ rotor, spun at $4000 \mathrm{~Hz}$, and maintained at $4^{\circ} \mathrm{C}$ to minimize sample degradation. Preparation and handling of rat liver tissue samples in this way have been documented by Waters et al. (2000), who reported that high-resolution MAS MRS of frozen rat liver samples had no significant differences compared with fresh liver.

For each sample, a pulse collect spectrum was first acquired using TR $=20 \mathrm{~s}$ and a $45^{\circ}$ excitation pulse. Sixteen data collects were summed over a sweep width of $6000 \mathrm{~Hz}$ into 32 $\mathrm{K}$ data points. These data were used to calculate the contribution of lipid- $\mathrm{CH}_{2}$ relative to the total spectral integral ( -0.5 to $10 \mathrm{ppm})$.

A pulse collect MR spectrum was then obtained with presaturation of the water resonance, using TR $=5 \mathrm{~s}$, a $45^{\circ}$ excitation pulse, 64 averages, and a sweep width of $6000 \mathrm{~Hz}$. These data were used to determine the tCho levels as a percentage of total spectral integral (excluding the region of residual water signal at 4.8-5.2 $\mathrm{ppm}$ ).

Finally, a Carr-Purcell-Meiboom-Gill (CPMG) sequence TR - $\left[90_{x}-\left(\mathrm{T} 180^{\circ}{ }_{y}-\mathrm{T}\right)_{\mathrm{n}}\right]$-collect free-induction-decay (FID) (with water suppression) was applied to minimize the contribution of broad short $T_{2}$ resonances using TR $=5 \mathrm{~s}, \mathrm{~T}=2 \mathrm{~ms}, n=40$, and 128 averages. Data were collected into $32 \mathrm{~K}$ data points with a sweep width of $6000 \mathrm{~Hz}$ and a $T_{2}$ relaxation delay of $80 \mathrm{~ms}$. These spectra provided estimates of glycerophosphocholine and phosphocholine (GPC/PC) levels relative to the total spectral integral (excluding the region of residual water signal at 4.8-5.2 ppm).

The peak areas of the MR spectra from intact liver tissue were processed using KnowItAll Informatics System v7.8 Software (Bio-Rad Laboratories, Hertfordshire, U.K.). The MR spectra were automatically phased, and additional manual phasing was performed only if automatic phasing was not optimal. Baseline correction using the automatic spline algorithm 
within Knowit-All was applied. Peaks were assigned in accordance with databases from published material on nuclear magnetic resonance of rat liver (Bollard et al., 2000). The peaks of interest were assumed to be sufficiently separated for integration of a "bin" around the resonance and were considered to be a close surrogate of the true peak integral, as previously published (Cobbold et al., 2009). The spectral integral of each individual region was measured, in addition to the total spectral integral $(0-10 \mathrm{ppm})$ excluding the region 4.8$5.2 \mathrm{ppm}$ of the residual water peak in sequences in which water suppression was applied (Cobbold et al., 2009). Metabolite levels were expressed as percentages relative to the total spectrum integral from which they were measured. Normalizing to the total integral eliminated the effect of increases in signal-to-noise due to differing sample mass.

\section{Statistical analyses}

In vivo MR data were compared using a repeated measures ANOVA to determine if significant differences were present between ANIT and PFCs. Post hoc Bonferroni analysis was then performed to establish significant changes in each group. Ex vivo MAS MRS data and all other terminal samples were compared using a one-way ANOVA. Significance was considered to be reached at the $p<0.05$ level, and results are quoted as mean $\pm \mathrm{SE}$.

\section{RESULTS}

\section{Body Weight}

A one-way ANOVA showed that the PFC_groups had significantly different body weights $(p=0.02)$. Bonferroni post hoc tests showed animals in the ANIT_0.05\% and ANIT_0.04\% groups experienced a reduced weight gain, relative to their PFCs at weeks $1(p<0.0001)$ and $2(p<0.01)$ despite similar food intake (Table 1). A one-way ANOVA showed ANIT_ $0.025 \%$ group had a significantly higher body weight relative to the higher doses (ANIT_0.025\% $221 \pm 3 \mathrm{~g}$, ANIT_0.04\% $186 \pm 4 \mathrm{~g}$ [ $p<0.05]$, and ANIT_0.05\% $172 \pm 4 \mathrm{~g}$ $[p<0.05]$ ). ANIT-fed groups had $<10 \%$ variation in weights within each group (data not shown), this indicates similar individual food intake within each group, and hence similar ingested ANIT dose within each group. This variation in weight was also true for PFCs.

\section{Food Intake}

A dose-dependent reduction in appetite was apparent with increasing ANIT dose. Food intake was $120 \pm 3,110 \pm 3$, and $92 \pm 5 \mathrm{~g}$ for ANIT_0.025\%, ANIT_0.04\%, and ANIT_0.05\%, respectively, by the end of week 2 (Table 1). Average ANIT ingestion (calculated from the average for weeks 1 and 2) was calculated to be 32.0,43.5, and $47.1 \mathrm{mg}$ for the ANIT_0.025\% group, ANIT_0.04\% group, and ANIT_0.05\% group, respectively, over the 14-day period. These data corresponded to an average daily dosage 9.7, 16.9, and $19.1 \mathrm{mg} / \mathrm{kg}$ of body weight (in the same group order), respectively.

\section{In Vivo ${ }^{31} \mathrm{P}$ MRS Findings}

In vivo MR spectral quality was substandard in three animals studied due to inadequate signal to noise or abnormal phasing. As two data sets were incomplete for both the PFC_0.025\% and ANIT_0.025\%, only $n=8$ animals were subsequently analyzed for each group. Numbering the animals 1-10 in order of the initial in vivo MR study, the first eight complete MR data sets were included for each group. Samples for MAS MRS, sera, and histopathology were matched to the in vivo group for all but one animal in the PFC_0.025\% group.

Representative unlocalized ${ }^{31} \mathrm{P}$ MR spectra from the control and ANIT_0.05\% groups are shown in Figure 1, which comprise resonances of hepatic origin and also a small overlapping contribution of resonances from overlying muscle. The PME region comprises 
of contributions from a number of resonances, including PC, phosphoethanolamine (PE), and various sugar phosphates. Similarly, the PDE resonance appears broad, due to its multicomponent nature, and includes GPC and glycerophosphoethanolamine (GPE). A narrower prominent peak originates from $\mathrm{PCr}$ in the abdominal wall muscle, overlying the liver. Owing to the purely nonhepatic origin of the PCr resonance, this was not included in the measurement of the total phosphorus signal level (tPh).

The ratios of PDE/tPh at baseline, week 1, and week 2 are shown in Figure 2 for all groups. A repeated measures ANOVA showed that both the ANIT_0.05\% group and ANIT_0.04\% group had significantly different levels of PDE at weeks 1 and $2(p=0.045$ and $p=0.02$, respectively). The $\mathrm{PDE} / \mathrm{tPh}$ remained similar throughout the supplemented period between the ANIT_0.025\% and its PFC group. Bonferroni post hoc tests showed no significant differences in baseline PDE/tPh levels for any ANIT-fed group relative to its PFC group, however, levels were quite variable. Prior to baseline animals were fed ad lib, differing intake of "normal feed" may possibly affect the PDE ratio. However, it is more probable that the variation is mainly attributed to the fitting of spectra when animals at baseline are scanned. Given the smaller size of the animal at baseline, the shimming of each was more difficult and detrimental to spectral resolution and hence reproducibility of metabolites such as PDE at relatively low concentrations. Baseline PME showed less variability in linewidths and therefore were more readily fitted at baseline (and at weeks 1 and 2).

A repeated measures ANOVA test showed that PME was also significantly different in the ANIT_0.05\% group $(p=0.002)$. At baseline, all groups had similar PME/tPh levels $(p>$ 0.05). Post hoc Bonferroni tests showed that PME was significantly different in the ANIT_0.05\% group at week $1(p<0.05)$ and at week $2(p<0.01)$ (Fig. 2). The increase in $\mathrm{PME} / \mathrm{tPh}$ was not seen in the lower two doses.

$\mathrm{PCr} / \mathrm{tPh}$ ratios were similar in all ANIT-fed relative to PFC groups for all doses at baseline, week 1 , and week 2 ( $<0.25$ which is approximately $25 \%$ of the total spectrum integral), which corroborates the assumption that the MR signal contribution from the abdomen was comparable between PFC- and ANIT-fed groups.

\section{Clinical Chemistry}

After 2 weeks of ANIT feeding, all ANIT-fed groups showed a significant increase in serum TBil $(p<0.0001)$ and ALP relative to PFC groups $(p<0.003$, Table 1$)$. A one-way ANOVA test confirmed TBil increased in a dose-dependent manner $(p<0.0001)$. ALB levels were significantly decreased at the two higher ANIT doses (ANIT_0.05\% and ANIT_0.04\%).

The ANIT_0.05\% group was the only experimental group that also showed concomitant increases in ALT and AST relative to PFC $(p<0.05)$. Comparison of Chol levels showed that ANIT-fed animals were significantly different to controls $(p=0.01)$. Post hoc tests showed that Chol was only significantly different in the ANIT_0.04\% animals, relative to PFCs, although the ANIT_0.05\% group did exhibit increased levels that approached significance $(p=0.07)$.

\section{Histopathology}

Microscopically, peribiliary hyperplasia, inflammation, and fibrosis were observed in all ANIT-treated animals (Table 2, Fig. 3). A dose-related increase in the severity of biliary hyperplasia with increasing doses of ANIT was apparent. All animals treated with 0.025 and $0.04 \%$ ANIT had mild to moderate hyperplasia (grades 2 and 3), and animals treated with $0.05 \%$ ANIT had mild to marked hyperplasia (grades 2-4). There was no clear dose-related change in severity for the peribiliary inflammation or fibrosis. 
In addition to the changes in the biliary tract, there was a dose-related increase in incidence and severity of focal hepatocellular necrosis in animals treated with ANIT. The ANIT_0.05\% group showed the highest incidence of moderate hepatocellular necrosis.

\section{Ex Vivo ${ }^{1} \mathrm{H}$ MAS MRS Findings}

All ANIT-fed groups showed a reduced \% lipid- $\mathrm{CH}_{2}$ relative to PFCs in the pulse collect spectrum as illustrated in Figure $4(p=0.0014)$. However, the contribution of lipid- $\mathrm{CH}_{2}$ to the total spectral integral did not vary significantly with ANIT dosage $(p>0.05)$.

A one-way ANOVA on the relative percentage of tCho confirmed that it was increased in the ANIT-fed animals $(p<0.0001)$. Post hoc tests identified this only reached significance in the ANIT_0.05\% group relative to its PFC. A visible increase is seen in Figures $4 \mathrm{c}$ and $4 \mathrm{~d}$. Unlike the PFC groups, the ANIT-fed groups also had significantly different responses in tCho confirming a dose-dependent response independent of diet $(p<0.0001)$. Further analysis of this region in the CPMG spectra suggested that the relative increase in tCho was consistent with increased levels of GPC + PC (Table 1 and Fig. 4). Although relative tCho levels were not significantly different in the ANIT_0.04\% group compared with the respective $\mathrm{PFC}$ group, a relative increase in $\mathrm{GPC}+\mathrm{PC}$ was observed in the CPMG data sets $(p<0.05)$. At the lower ANIT dose (ANIT_0.025\%), neither tCho nor GPC + PC were significantly elevated.

The Glc/Glyc was significantly different between ANIT-fed groups $(p<0.0014)$. Post hoc tests identified the ANIT_0.05\% group as the only group to show a significant increase in the relative Glc/Glyc levels relative to its PFC $(p<0.0001)$.

\section{DISCUSSION}

This study has shown that in vivo hepatic ${ }^{31} \mathrm{P}$ MR changes were detected within 1 week of chronic ANIT feeding with ANIT_0.05\% and ANIT_0.04\%. Changes in hepatic MRS biomarkers persisted with continued ANIT ingestion. Increased PDE/tPh was observed in vivo, consistent with elevated GPC + PC measured ex vivo using ${ }^{1} \mathrm{H}$ MAS MRS, in the presence of histological biliary damage and abnormal clinical chemistry. Increased PME/tPh and increased relative tCho reached significance in the ANIT_0.05\% group and correlated with more severe hepatic damage as demonstrated by elevated ALT and AST and biliary hyperplasia. Increased incidence of histologically observed marked hepatocellular necrosis was also observed in this group. Milder hepatobiliary damage resulting from the ANIT_0.025\% dose was not detectable in vivo using ${ }^{31} \mathrm{P}$ MRS. All ANIT groups could be distinguished from PFCs by significantly lower lipid levels.

\section{PDE Findings in Hepatic In Vivo ${ }^{31} \mathrm{P}$ MR Studies}

The elevation in hepatic PDE levels is consistent with ANIT-induced cell degeneration. This arises from phospholipids (a byproduct of cell membrane degeneration) and an increased contribution from phospholipids in bile during hepatocellular injury and cholestasis. This is consistent with previous reports, which found that intrahepatic cholestasis is a result of the sloughing of necrotic biliary epithelial cells into duct lumen following ANIT treatment (Lopez and Mazzanti, 1955; Tjandra et al., 2000). Similar increases in PDE in chronic ductopenic rejection have been reported, a disease that also has significant cholestasis and hepatocellular injury (Taylor-Robinson et al., 1998). Histological findings in our study found the presence of moderate-marked biliary hyperplasia and mild peribiliary inflammation corroborating with biliary dysfunction.

The contributions to the PDE resonance in ${ }^{31} \mathrm{P}$ MR spectra can be separated as GPC and GPE. The choline peak of the GPC resonance can be readily identified as a singlet in ${ }^{1} \mathrm{H}$ MR 
spectra, albeit overlapping with other choline-containing compounds. The ethanolamine resonance is a complex peak and more difficult to identify. The PDE changes were consistent with increases in measurements of GPC + PC at the two higher doses $(0.04$ and $0.05 \%$ ANIT). This was evident in the ANIT_0.04\% group in the absence of an increase in PME. This is consistent with previous studies, which reported increased GPC + PC with ANIT dosing 1 week after a single dose. ANIT is reported to have dose-dependent effects, which perhaps explains the unchanged levels at the lower dose (Waters et al., 2001).

The elevated GPC/PC levels we observed were also consistent with increased cell degeneration, leading to production of GPC, which is reflected in the PDE measurements. Another mechanism that could be responsible for elevated PC + GPC is obstruction of bile flow. Such cholestasis leads to accumulation of normal biliary components, such as PC and GPC in hepatocytes.

Further evidence for the correlation between the increase in PDE and the blockage of bile ducts is the subsequent elevations that we observed in ALP and TBil, measured at the time of hepatocyte and cholangiocyte injury. These serum changes are nonspecific but were apparent even at the low dose of $0.025 \%$ ANIT. Blood cholesterol levels were altered in ANIT-fed groups $(p<0.05$ for ANIT_0.04\%). The accumulation of cholesterol may arise from impaired bile flow, as biliary excretion is the major route for regulating hepatic cholesterol levels (Dietschy and Wilson, 1970).

Hence, we can conclude that the increase in PDE is consistent with blockage of bile ducts in addition to biliary hyperplasia. An increase in GPC would also be consistent with this, although a concomitant increase in PC cannot be completely ruled out.

\section{PME Findings in Hepatic In Vivo ${ }^{31} \mathrm{P}$ MR Studies}

The increase in PDE/tPh was accompanied by an increase in PME/tPh in the ANIT_0.05\% group. All ANIT-fed animals expressed a generally increased PME level at week 1, but only the ANIT_0.05\% group did not return to control levels by 2 weeks of ANIT feeding. The elevated PME correlated with a significant increase in tCho at 2 weeks. This is in accordance with increases in tCho seen at week 1 in single-dose ANIT studies (Waters et al., 2001). Again, this distinction was reflected in clinical chemistry, where substantial disruption to hepatocellular integrity was indicated by increases in transaminases, not seen at the lower two doses (Kew, 2000). ALT and AST increases correlate with the leakage of these enzymes from damaged hepatocytes and their cell membranes (Rochling, 2001). In addition to increased ALT and AST, raised TBil levels were observed in the higher dose group relative to the other ANIT and PFC groups (more than three times the ANIT_0.04\% levels $p<0.05$ ), suggesting that in addition to intrahepatic cholestasis, there is the presence of increased severity of ANIT-induced hepatocyte necrosis (as confirmed by ALT and AST) at the higher dose (Rochling, 2001). Kossor et al. (1995) found that the hepatocyte response to severe hepatocellular damage was subsequent hepatocellular proliferation. However, histopathology in our study showed moderate hepatocellular damage in addition to marked biliary hyperplasia, but with no evidence of hepatocellular proliferation.

In contrast, we did note significant biliary hyperplasia in the ANIT_0.05\% group, which may underlie the significant PME changes in vivo. Hence, it may be hypothesized that the increase in $\mathrm{PME} / \mathrm{tPh}$ observed in vivo was indicative of moderate hepatocellular necrosis, following severe hepatic damage as characterized by AST and ALT, in addition to the moderate biliary injury (Kossor et al., 1995). Bile duct proliferation was also documented by Robertson et al. (2000), when using $10 \mathrm{mg} / \mathrm{kg}$ ANIT. This is supported by the raised tCho, which has been suggested to be due to biliary hyperplasia, as a result of severe cholestasis (Waters et al., 2001). In our study, we found the concomitant hepatocellular necrosis and 
bile duct proliferation in the ANIT_0.05\% group caused by these processes is also reflected ex vivo by a significant increase in the tCho region.

It must be noted that although the changes in AST in ANIT animals were different to controls, the values for PFC animals are much higher than those quoted in the literature, $\sim 100 \mathrm{U} / 1$, almost half the amount we measured. This increase may result from restricted dietary intake as caloric restriction has been shown to increase transaminase enzyme activities including AST in mice (Hagopian et al., 2003).

It is noteworthy that overall at 14 days, both ex vivo and in vivo MRS are sensitive enough to differentiate the ANIT_0.04\% from the ANIT_0.05\% by increases in tCho and PME/tPh. However, given the biliary hyperplasia and focal liver necrosis are dynamic processes, attributing the rise in the multicomponent PME resonance to a single process is not possible in this model.

The effect on PME/tPh was not demonstrated in the ANIT_0.04\% or ANIT_0.025\% groups, supporting a dose-dependent response to ANIT. Thus, biliary hyperplasia together with hepatocellular necrosis is only observed at sufficiently high doses, leading to PME increases.

\section{Lipid Changes by Ex Vivo ${ }^{1} \mathrm{H}$ MAS MRS}

A decrease in triglycerides was also observed in ANIT-fed groups. Again, this effect has been reported at 1 week postdose in a previous single-dose study (Waters et al., 2001, 2002). The decrease in lipid triglyceride suggests disrupted lipid metabolism, potentially due to impaired hepatic lipogenesis, as a result of hepatic dysfunction.

\section{Glucose/Glycogen Changes by Ex Vivo ${ }^{1} \mathrm{H}$ MAS MRS}

Glc/Glyc levels were generally increased in all ANIT-fed groups and reached significance in the ANIT_0.05\% group. The changes in Glc/Glyc recorded suggest perturbed glucose metabolism by continuous chronic ingestion of ANIT thought to arise from a reduced hepatic gluconeogenesis and glycolysis due to hepatobiliary injury. Similar findings in urinary glucose have been observed at 1 week in single ANIT dose experiments (BeckwithHall et al., 1998; Waters et al., 2001,2002).

\section{Limitations}

MRS protocol—Here, we implement a nonlocalized method for MRS. Methods for localization of fast-relaxing nuclei are available but may well be susceptible to motion (e.g., image-selected in vivo spectroscopy [ISIS]) or lose signal during localization (stimulated echo acquisition mode[STEAM]). Given the bigger voxels that can be used in the clinic (a good spectrum can be achieved in $<15 \mathrm{~min}$ for a $6 \times 6 \times 6 \mathrm{~cm}$ voxel in humans), the signal loss during STEAM can be easily overcome in the clinic and would give a more specific measure of hepatic phospholipid metabolism. The detection of PCr can be taken as a measure of muscle contamination. Here, we tested that this contribution was constant.

The effect of not using respiratory gating may affect the results, as each pulse is not exciting the exact same area. However, given the 45-min scan time and the large number of averages, the effective area sampled can be considered to be similar over all groups. Respiratory triggering may have reduced the variation in measures of PDE and PME through more accurate localization, reduction in frequency drift, and better shimming which would improve spectral resolution. Given the larger voxel size, in vivo using respiratory gating together with ISIS would be recommended. 
Handling and preparation of rat serum—Rat serum samples were frozen before clinical chemistry and analyzed within 7 days of sample receipt. No sample was analyzed after repeated freeze-thaw cycles. This may in some cases potentially lead to freeze-thaw artifacts or changes but has been shown in-house at GlaxoSmithKline to have a minimal effect on rat serum analyte levels after freezing at $-80^{\circ} \mathrm{C}$ and is therefore part of the standard operating procedures. Levels of ALP, ALT, and AST from frozen sera have been reported to be similar to fresh sera in canines (Reynolds et al., 2006), and tests on the cholesterol levels in sera from humans show little change on freezing (Comstock et al., 2001), hence we have assumed here that levels of these markers will remain similarly unchanged on freezing in rat sera. Levels of infant TBil, however, have been reported to change when frozen at $-80^{\circ} \mathrm{C}$ (21\% increase) after 1 month (Amin et al., 2008). However, all samples between groups were treated in the same way, therefore changes due to the handling of samples can be assumed to have the same effect in the PFC and ANIT animals.

Specificity of changes-To establish more specific markers for biliary hyperplasia, which is a particular area of interest in preclinical studies of drug toxicity, more precise models of biliary damage, which do not cause hepatocellular damage, need to be validated. In addition, histopathological and clinical chemistry correlates at earlier ex vivo time points would add more information about the complex dynamics of the pathologies seen by 14 days.

For more detailed clarification of the complex metabolites seen using MRS techniques in acute liver injury, the ex vivo extracts could be run at higher magnetic field strengths, whereas the in vivo ${ }^{31} \mathrm{P}$ MRS could be undertaken on MR systems where the resolution is improved to resolve PC and GPC separately.

This study considered the effects of a single hepatotoxin ANIT, and hence, the sensitivity of ${ }^{31} \mathrm{P}$ MRS to biliary hyperplasia and hepatocellular damage seen in animal models with this drug. It would be important to carry out studies on a range of hepatotoxins preclinically to assess the potential ability of ${ }^{31} \mathrm{P}$ MRS to distinguish the effects of different toxins. With the use of more sophisticated localized techniques, the spectroscopic voxel could be placed to focus on just bile ducts or the main liver lobes allowing discrimination of the effect of hepatotoxins on either site.

\section{Role of MRS in Hepatotoxicity}

Existing biomarkers such as TBil and ALP are both sensitive to the severity of liver damage and show a dose-response to ANIT ingestion. However, neither of these is liver specific with TBil rising in hemolysis and ALP from bone (and placenta) while ${ }^{31} \mathrm{P}$ MRS is localized to the liver. Hepatic ${ }^{31} \mathrm{P}$ MRS can add a more noninvasive measure here, which has the potential to give dose/severity-dependent measures of cellular dynamics. The implementation of a sensitive measure of PDE and PME in the clinic will provide an insight into cellular membrane metabolism and cellular proliferation and necrosis, which is not given by serum measures such as ALP and TBil.

Both ${ }^{1} \mathrm{H}$ MAS MRS and clinical chemistry have been employed here as complementary techniques to corroborate in vivo ${ }^{31} \mathrm{P}$ MRS. Both the former techniques still provide useful sensitive measures alongside the ${ }^{31} \mathrm{P}$ MRS, such as liver enzymatic activity and hepatic lipid changes. These can also be achievable in the clinic with perhaps the less invasive ${ }^{1} \mathrm{H}$ MRS in vivo replacing the need for liver biopsies in ${ }^{1} \mathrm{H}$ MAS MRS.

Here, we have detected changes in in vivo ${ }^{31} \mathrm{P}$ MRS, ex vivo ${ }^{1} \mathrm{H}$ MAS MRS, and clinical chemistry, in response to ANIT toxicity, all of which were corroborated with histology. With the capability of localized ${ }^{31} \mathrm{P}$ MRS, this technique has the potential to provide 
noninvasive site-specific dynamic information on cellular membrane metabolism. Changes in PDE and PME are indicative of modifications in cell death and regeneration, cell membrane synthesis and breakdown, and the associated proliferation of cells. Unlike traditional endpoints of hepatotoxicity, localized ${ }^{31} \mathrm{P}$ MRS can be liver specific, hence markers are direct measures of the diseased site and are repeatable from the same tissue. Plasma clinical chemistry markers are systemic and are not necessarily organ specific. However, given their ease and the additional information, they provide there would be benefits in using such assays in conjunction with ${ }^{31} \mathrm{P}$ MRS in toxicity evaluation trials. ${ }^{1} \mathrm{H}$ MAS MRS data from liver samples can also provide a wealth of information on hepatic lipids as well as more specific information on choline but are potentially subject to sampling variability within the liver. Also, although ${ }^{1} \mathrm{H}$ MAS MRS corroborates the ${ }^{31} \mathrm{P}$ MRS and has a high sensitivity, it is not a comparable test as it is performed on ex vivo samples. Lower resolution ${ }^{1} \mathrm{H}$ MR spectra obtained in vivo can avoid sampling variability, and localized ${ }^{1} \mathrm{H}$ MRS of the liver can be conducted during the same MRI examination to give information on hepatic lipid and choline in response to drug treatment, in addition to cellular dynamics from ${ }^{31} \mathrm{P}$ MRS, and in conjunction with serum measurements of hepatic enzymatic activity and bilirubin.

\section{CONCLUSIONS}

We have shown that in vivo ${ }^{31} \mathrm{P}$ MRS and ex vivo ${ }^{1} \mathrm{H}$ MAS MRS have the potential to characterize hepatobiliary dysfunction. In our study, severe ANIT-induced hepatic and biliary damage can be characterized noninvasively in vivo using ${ }^{31} \mathrm{P}$ MRS. An increase in $\mathrm{PDE}$ and an increase in GPC + PC ex vivo were noted when biliary hyperplasia was present, in addition to parenchymal inflammation and hepatocellular damage. Using MRS, markers sensitive to severity of disease can be followed longitudinally. Further investigations are needed to determine the specificity of the PDE change, relative to biliary hyperplasia, and PME in relation to hepatocellular damage.

\section{Acknowledgments}

The MRUI software package was kindly provided by the participants of the EU Network programmes: Human Capital and Mobility, CHRX-CT94-0432 and Training and Mobility of Researchers, ERB-FMRX-CT970160. J.F.L.C., S.D.T.-R., J.D.B., and I.J.C. are grateful to the NIHR Biomedical Research Facility at Imperial College London for providing infrastructure support.

FUNDING: GlaxoSmithKline Pharmaceuticals (GSK 3445).

\section{REFERENCES}

Amin SB, Ahlfors CE. Bilirubin-binding capacity in premature infants. Pediatrics. 2008; 121:872-873. [PubMed: 18381560]

Angus PW, Dixon RM, Rajagopalan B, Ryley NG, Simpson KJ, Peters TJ, Jewell DP, Radda GK. A study of patients with alcoholic liver-disease by P-31 nuclear magnetic-resonance spectroscopy. Clin. Sci. 1990; 78:33-38. [PubMed: 2153493]

Balazs T, McLaughlan JM, Grice HC, Murray TK. Hepatic tests in toxicity studies on rats. Toxicol. Appl. Pharmacol. 1961; 3:71-79. [PubMed: 13686221]

Becker BA, Plaa GL. Nature of alpha-naphthylisothiocyanate-induced cholestasis. Toxicol. Appl. Pharmacol. 1965; 7:680-685. [PubMed: 5866807]

Beckwith-Hall BM, Nicholson JK, Nicholls AW, Foxall PJD, Lindon JC, Connor SC, Abdi M, Connelly J, Holmes E. Nuclear magnetic resonance spectroscopic and principal components analysis investigations into biochemical effects of three model hepatotoxins. Chem. Res. Toxicol. 1998; 11:260-272. [PubMed: 9548796] 
Bell JD, Cox IJ, Sargentoni J, Peden CJ, Menon DK, Foster CS, Watanapa P, Iles RA, Urenjak J. A $\mathrm{P}-31$ and H-1-NMR investigation in vitro of normal and abnormal human liver. Biochim. Biophys. Acta. 1993; 1225:71-77. [PubMed: 8241291]

Bollard ME, Garrod S, Holmes E, Lindon JC, Humpfer E, Spraul M, Nicholson JK. High-resolution (1)H and (1)H-(13)C magic angle spinning NMR spectroscopy of rat liver. Magn. Reson. Med. 2000; 44:201-207. [PubMed: 10918318]

Changani KK, Davidson BR, Bell JD, Taylor-Robinson SD, Fuller BJ. A comparison of ATP recovery or phosphomonoester inorganic phosphate ratio to assess metabolic activity in liver at hypothermia: A P-31 NMR study. Cryo. Lett. 1998; 19:183-188.

Cobbold JFL, Anstee QM, Goldin RD, Williams HRT, Matthews HC, North BV, Absalom N, Thomas HC, Thursz MR, Cox RD, et al. Phenotyping murine models of non-alcoholic fatty liver disease through metabolic profiling of intact liver tissue. Clin. Sci. 2009; 116:403-413. [PubMed: 18694396]

Comstock GW, Burke AE, Norkus EP, Gordon GB, Hoffman SC, Helzlsouer KJ. Effects of repeated freeze-thaw cycles on concentrations of cholesterol, micronutrients, and hormones in human plasma and serum. Clin. Chem. 2001; 47:139-142. [PubMed: 11148194]

Corbin IR, Buist R, Peeling J, Zhang M, Uhanova J, Minuk GY. Hepatic P-31 MRS in rat models of chronic liver disease: Assessing the extent and progression of disease. Gut. 2003; 52:1046-1053. [PubMed: 12801965]

Dahm LJ, Schultze AE, Roth RA. An antibody to neutrophils attenuates alpha-naphthylisothiocyanateinduced liver injury. J. Pharmacol. Exp. Ther. 1991; 256:412-420. [PubMed: 1846424]

Dietschy JM, Wilson JD. Regulation of cholesterol metabolism. N. Engl. J. Med. 1970; 282:11791183. [PubMed: 4985822]

Goldfarb S, Singer EJ, Popper H. Experimental cholangitis due to alpha-naphthyl-isothiocyanate (ANIT). Am. J. Pathol. 1962; 40:685-698. [PubMed: 13899749]

Hagopian K, Ramsey JJ, Weindruch R. Caloric restriction increases gluconeogenic and transaminase enzyme activities in mouse liver. Exp. Gerontol. 2003; 38:267-278. [PubMed: 12581790]

Indacoch N, Plaa GL. Functional effects of alpha-naphthylisothiocyanate in various species. Toxicol. Appl. Pharmacol. 1971; 19:71-80. [PubMed: 5570568]

Jalan R, Cox IJ, Wilson J, AlaKorpela M, Schweiso J, Bell JD. In vivo hepatic phosphorus-31 magnetic resonance spectroscopy after infusion of L-alanine: Understanding the biochemical basis of hepatic decompensation. Hepatology. 1996a; 24:57.

Jalan R, Sargentoni J, Bryant DJ, Coutts GA, Bell JD, Cox IJ, Rolles K, Burroughs AK, TaylorRobinson SD, Steiner RE. Assessment of in vivo hepatic P-31 magnetic resonance spectroscopy as a prognostic marker in patients with primary biliary cirrhosis. Hepatology. 1995; 22:68.

Jalan R, Sargentoni J, Coutts GA, Bell JD, Rolles K, Burroughs AK, Taylor-Robinson SD. Hepatic phosphorus-31 magnetic resonance spectroscopy in primary biliary cirrhosis and its relation to prognostic models. Gut. 1996b; 39:141-146. [PubMed: 8881826]

Jalan R, Taylor-Robinson SD, Hodgson HJF. In vivo hepatic magnetic resonance spectroscopy: Clinical or research tool? J. Hepatol. 1996c; 25:414-424. [PubMed: 8895024]

Kew MC. Serum aminotransferase concentration as evidence of hepatocellular damage. Lancet. 2000; 355:591-592. [PubMed: 10696975]

Kossor DC, Goldstein RS, Ngo W, Denicola DB, Leonard TB, Dulik DM, Meunier PC. Biliary epithelial-cell proliferation following alpha-naphthylisothiocyanate (ANIT) treatmentRelationship to bile-duct obstruction. Fundam. Appl. Toxicol. 1995; 26:51-62. [PubMed: 7657062]

Krell H, Hoke H, Pfaff E. Development of intra-hepatic cholestasis by alpha-naphthylisothiocyanate in rats. Gastroenterology. 1982; 82:507-514. [PubMed: 7054045]

Leonard TB, Popp JA, Graichen ME, Dent JG. Alpha-naphthylisothiocyanate induced alterations in hepatic drug-metabolizing-enzymes and liver morphology-Implications concerning anticarcinogenesis. Carcinogenesis. 1981; 2:473-482. [PubMed: 7273328]

Lopez M, Mazzanti L. Experimental investigations on alpha-naphthyl-iso-thiocyanate as a hyperplastic agent of the biliary ducts in the rat. J. Pathol. Bacteriol. 1955; 69:243-250. [PubMed: 13243192] 
Menon DK, Sargentoni J, Taylor-Robinson SD, Bell JD, Cox IJ, Bryant DJ, Coutts GA, Rolles K, Burroughs AK, Morgan MY. Effect of functional grade and etiology on in vivo hepatic P-31 magnetic resonance spectroscopy in cirrhosis-Biochemical basis of spectral appearances. Hepatology. 1995; 21:417-427. [PubMed: 7843715]

Munakata T, Griffiths RD, Martin PA, Jenkins SA, Shields R, Edwards RHT. An in vivo P-31 MRS study of patients with liver cirrhosis-Progress towards a noninvasive assessment of disease severity. NMR Biomed. 1993; 6:168-172. [PubMed: 8499248]

Naressi A, Couturier C, Devos JM, Janssen M, Mangeat C, de Beer R, Graveron-Demilly D. Javabased graphical user interface for the MRUI quantitation package. Magn. Reson. Mater. Phys. Biol. Med. 2001; 12:141-152.

Plaa GL, Priestly BG. Intrahepatic cholestasis induced by drugs and chemicals. Pharmacol. Rev. 1976; 28:207-273. [PubMed: 16281]

Reynolds B, Taillade B, Medaille C, Palenche F, Trumel C, Lefebvre HP. Effect of repeated freezethaw cycles on routine plasma biochemical constituents in canine plasma. Vet. Clin. Pathol. 2006; 35:339-340. [PubMed: 16967422]

Robertson DG, Reily MD, Sigler RE, Wells DF, Paterson DA, Braden TK. Metabonomics: Evaluation of nuclear magnetic resonance (NMR) and pattern recognition technology for rapid in vivo screening of liver and kidney toxicants. Toxicol. Sci. 2000; 57:326-337. [PubMed: 11006362]

Rochling FA. Evaluation of abnormal liver tests. Clin. Cornerstone. 2001; 3:1-12. [PubMed: 11501190]

Sequeira S, So PW, Everett JR, Elcombe CR, Kelvin AS, Nicholson JK. H-1-NMR spectroscopy of biofluids and the investigation of xenobiotic-induced changes in liver biochemistry. J. Pharm. Biomed. Anal. 1990; 8:945-949. [PubMed: 2100647]

Taylor-Robinson SD, Sargentoni J, Bell JD, Saeed N, Changani KK, Davidson BR, Rolles K, Burroughs AK, Hodgson HJF, Foster CS, et al. In vivo and in vitro hepatic P-31 magnetic resonance spectroscopy and electron microscopy of the cirrhotic liver. Liver. 1997; 17:198-209. [PubMed: 9298490]

Taylor-Robinson SD, Sargentoni J, Bell JD, Thomas EL, Marcus CD, Changani KK, Saeed N, Hodgson HJF, Davidson BR, Burroughs AK, et al. In vivo and in vitro hepatic phosphorus-31 magnetic resonance spectroscopy and electron microscopy in chronic ductopenic rejection of human liver allografts. Gut. 1998; 42:735-743. [PubMed: 9659173]

Tjandra K, Sharkey KA, Swain MG. Progressive development of a Th1-type hepatic cytokine profile in rats with experimental cholangitis. Hepatology. 2000; 31:280-290. [PubMed: 10655247]

Vanhamme L, van den Boogaart A, Van Huffel S. Improved method for accurate and efficient quantification of MRS data with use of prior knowledge. J. Magn. Reson. 1997; 129:35-43. [PubMed: 9405214]

Waters NJ, Garrod S, Farrant RD, Haselden JN, Connor SC, Connelly J, Lindon JC, Holmes E, Nicholson JK. High-resolution magic angle spinning H-1 NMR spectroscopy of intact liver and kidney: Optimization of sample preparation procedures and biochemical stability of tissue during spectral acquisition. Anal. Biochem. 2000; 282:16-23. [PubMed: 10860494]

Waters NJ, Holmes E, Waterfield CJ, Farrant RD, Nicholson JK. NMR and pattern recognition studies on liver extracts and intact livers from rats treated with [alpha]-naphthylisothiocyanate. Biochem. Pharmacol. 2002; 64:67-77. [PubMed: 12106607]

Waters NJ, Holmes E, Williams A, Waterfield CJ, Farrant RD, Nicholson JK. NMR and pattern recognition studies on the time-related metabolic effects of alpha-naphthylisothiocyanate on liver, urine, and plasma in the rat: An integrative metabonomic approach. Chem. Res. Toxicol. 2001; 14:1401-1412. [PubMed: 11599932] 


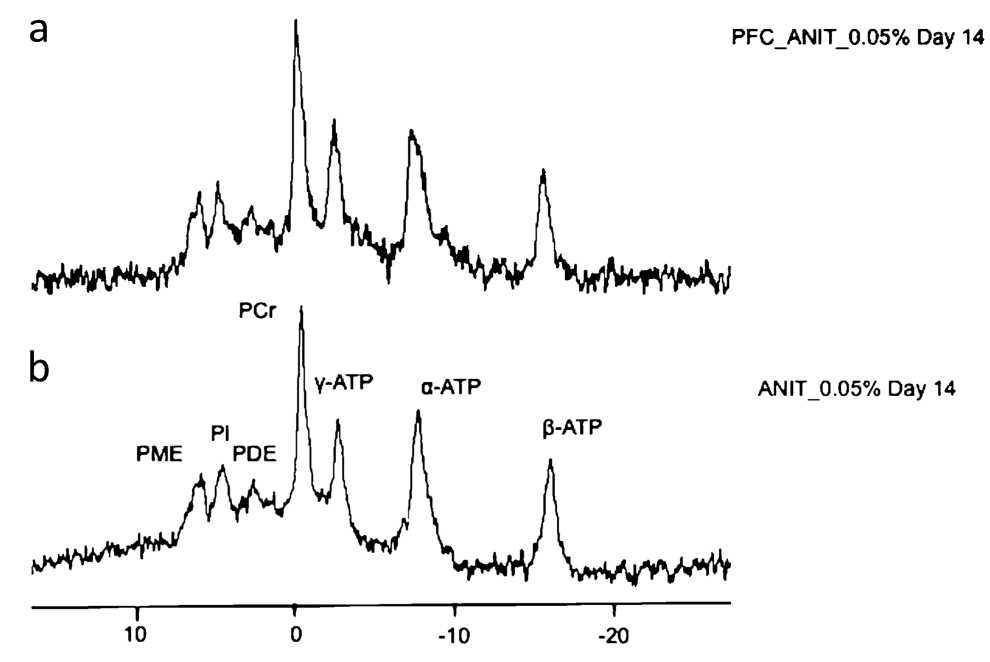

FIG. 1. Representative in vivo ${ }^{31} \mathrm{P}$ MR spectra from a rat liver after 2 weeks of ANIT supplemented diet and a PFC.

Resonances are assigned (from left to right) to PME, inorganic phosphate (Pi), PDE, phosphocreatine (PCr), and $\mathbf{\gamma}^{-}, \mathbf{a}-$-, and $\beta$-adenosine triphosphate. Above (a) PFC_0.05\% and below (b) ANIT_0.05\%. 
PDETTotal P
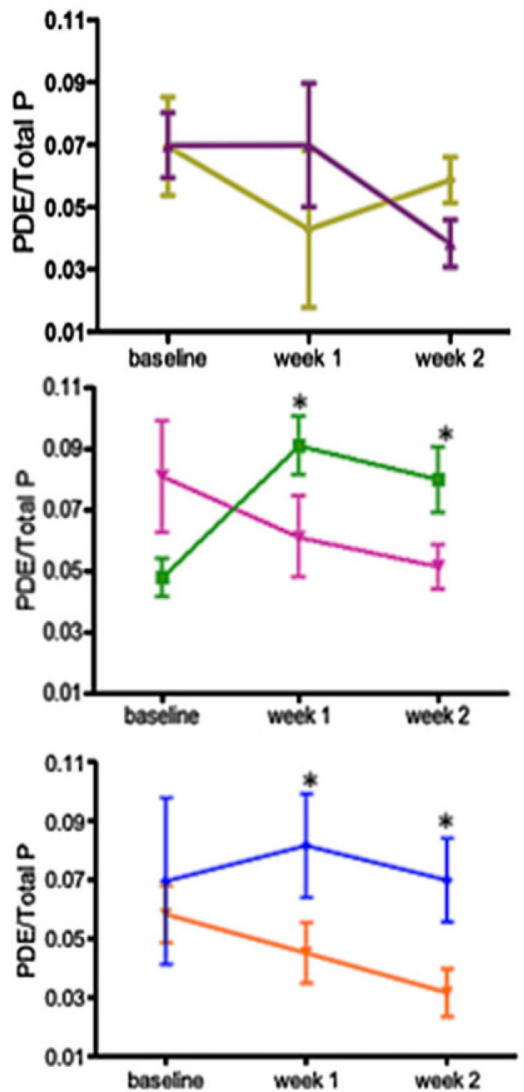

PME/Total P
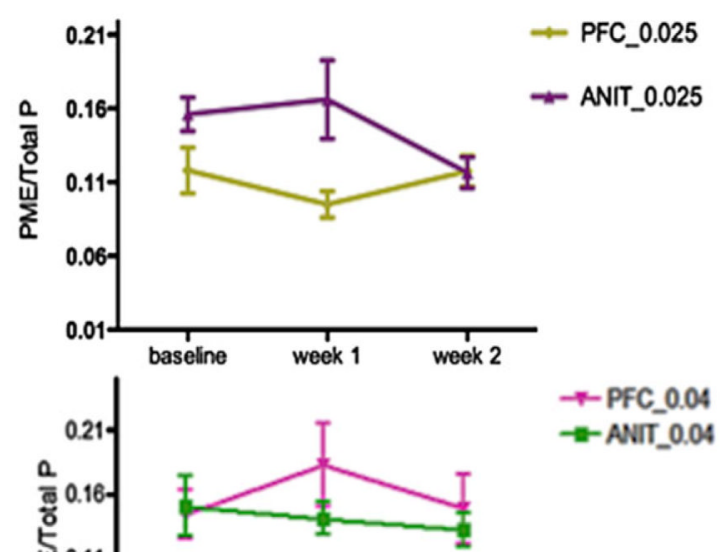

000
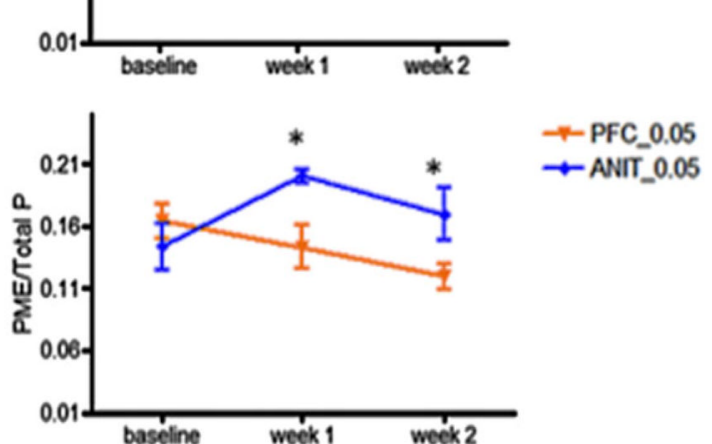

FIG. 2. Serial changes in PDE/tPh and PME/tPh for ANIT_0.025\%, ANIT_0.04\%, and ANIT_0.05\% groups and the corresponding PFC groups.

Ratios of PDE/Total phosphorus and PME/Total phosphorus for ANIT-fed groups with their corresponding PFC group at baseline, week 1 , and week 2 as measured by ${ }^{31} \mathrm{P}$ MR spectroscopy. 
a

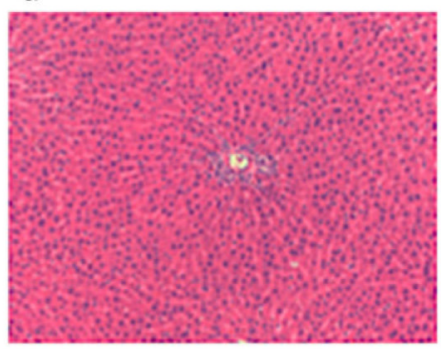

b

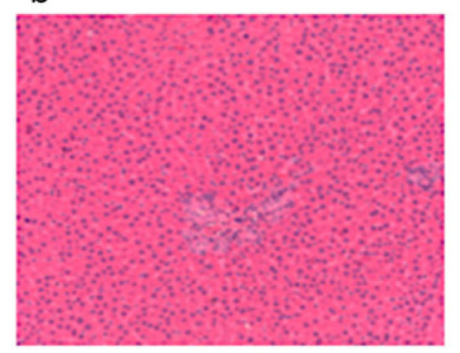

c

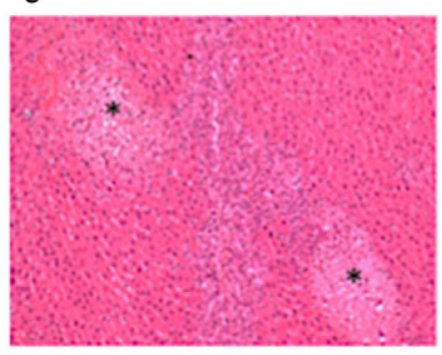

FIG. 3. (a-c) Representative histological sections from liver tissue from PFC_0.025\%, ANIT_0.025\%, and ANIT_0.05\% groups.

Sections are stained with hematoxylin and eosin and are shown at $\times 100$ magnification. (a) Liver from a control rat showing normal portal tract with small bile ducts and associated blood vessels. (b) Liver from an ANIT_0.025\% rat showing minimal biliary hyperplasia with a minimal peribiliary inflammatory cell infiltrate. (c) Liver from an ANIT_0.05\% animal. Areas of focal necrosis $(*)$ in hepatic parenchyma adjacent to portal area showing hepatobiliary hyperplasia, fibrosis, and peribiliary inflammation. 
PFC_0.05\%

a

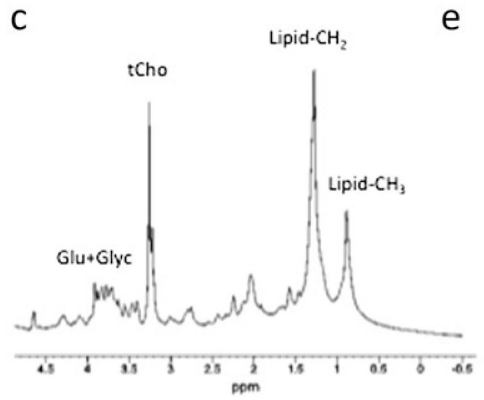

e
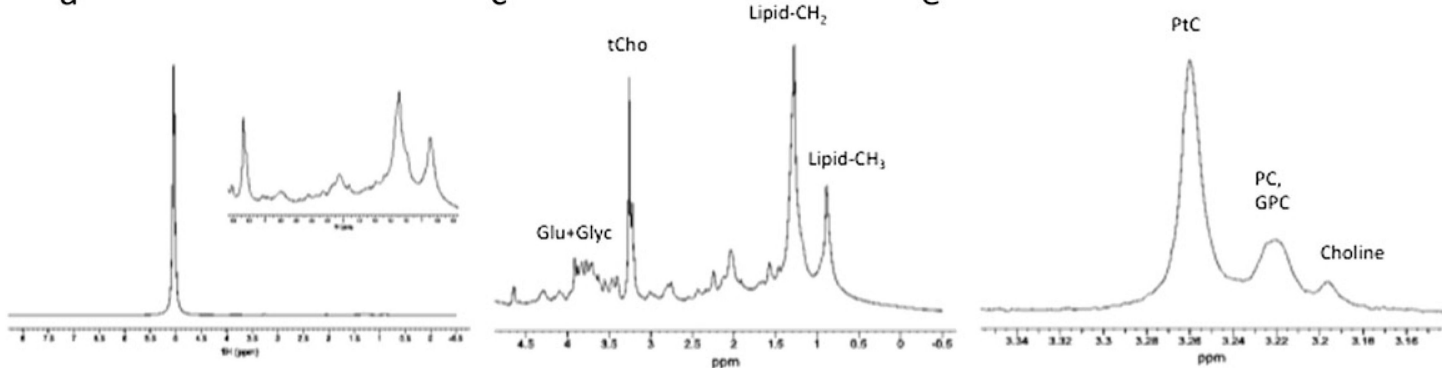

b

Lipid:Water

d

tCho

f

$P C+G P C$
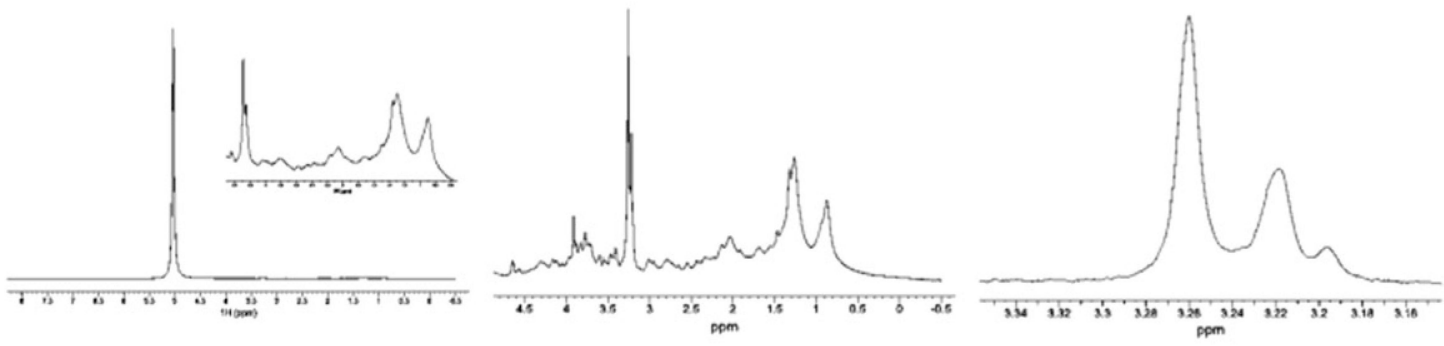

ANIT_0.05\%

FIG. 4. Illustrative ${ }^{1} \mathrm{H}$ MAS MR spectra of hepatic tissue from PFC_0.05\% (a, c, and e) compared with ANIT_0.05\% (b, d, and e).

The first panel ( $a$ and $b$ ) is an example of a pulse collect spectrum for both PFC_0.05\% (a) and ANIT_0.05\%. These spectra were used to calculate the water:lipid ratio. The insert shows the magnified lipid region. Decreased lipid peaks can be seen in the ANIT_0.05\% group. The second panel ( $\mathrm{c}$ and $\mathrm{d}$ ) is an example of a water-suppressed pulse collect spectrum for both PFC_0.05\% (c) and ANIT_0.05\% (d) used for calculating the total choline relative to total signal intensity. Increased choline can be seen in the ANIT_0.05\% group. The third panel (e and f) is an example of the water-suppressed CPMG spectrum used to get measures of PC + GPC and Glc/Glyc for both PFC_0.05\% (e) and ANIT_0.05\% (f). $\mathrm{PC}+\mathrm{GPC}$ is increased in the ANIT_0.05\% group. 
TABLE 1

Results for Food Intake, Weight, Clinical Chemistry, In Vivo ${ }^{31} \mathrm{P}$ MRS, Ex Vivo ${ }^{1} \mathrm{H}$ MAS MRS, and Clinical Chemistry for ANIT_0.025\%, ANIT_0.04\%, and ANIT_0.05\% and Corresponding PFC Groups at Day 14

\begin{tabular}{|c|c|c|c|c|c|c|}
\hline Group ( $n=8$ per group) & PFC_0.025\% & ANIT_0.025\% & PFC_0.04\% & ANIT_0.04\% & PFC_0.05\% & ANIT_0.05\% \\
\hline \multicolumn{7}{|l|}{ Phenotype } \\
\hline Total food intake $(\mathrm{g})$ & & $120 \pm 3$ & & $110 \pm 3$ & & $92 \pm 5$ \\
\hline Weight at 14 days (g) & $220 \pm 6$ & $221 \pm 3$ & $200 \pm 4$ & $186 \pm 4 t^{t}$ & $205 \pm 5$ & $172 \pm 4^{*}$ \\
\hline \multicolumn{7}{|l|}{ In vivo ${ }^{31} \mathrm{P} \mathrm{MRS}$} \\
\hline $\mathrm{PDE} / \mathrm{tPh}$ & $0.06 \pm 0.01$ & $0.04 \pm 0.01$ & $0.05 \pm 0.01$ & $0.08 \pm 0.01 \%$ & $0.03 \pm 0.01$ & $0.07 \pm 0.01^{*}$ \\
\hline $\mathrm{PME} / \mathrm{tPh}$ & $0.12 \pm 0.01$ & $0.12 \pm 0.01$ & $0.15 \pm 0.03$ & $0.13 \pm 0.01$ & $0.12 \pm 0.01$ & $0.17 \pm 0.02^{*}$ \\
\hline \multicolumn{7}{|l|}{ Ex vivo ${ }^{1} \mathrm{H}$ MAS MRS } \\
\hline $\begin{array}{l}\text { Lipid }\left(\mathrm{CH}_{2}\right) /(\text { total signal in pulse } \\
\text { collect spectrum }) \times 100 \%\end{array}$ & $0.99 \pm 0.05$ & $0.80 \pm 0.04^{\dagger}$ & $0.88 \pm 0.03$ & $0.75 \pm 0.02 \%$ & $0.92 \pm 0.02$ & $0.73 \pm 0.02^{*}$ \\
\hline $\begin{array}{l}\text { tCho/(total signal [excluding residual } \\
\text { water] in pulse collect spectrum with } \\
\text { water suppression }) \times 100 \%\end{array}$ & $6.14 \pm 0.50$ & $6.86 \pm 0.39$ & $5.85 \pm 0.27$ & $6.70 \pm 0.46$ & $6.34 \pm 0.22$ & $7.62 \pm 0.20^{*}$ \\
\hline $\begin{array}{l}\mathrm{PC}+\mathrm{GPC} /(\text { total signal [excluding } \\
\text { residual water] in CMPG sequence }) \times \\
100 \%\end{array}$ & $4.95 \pm 0.98$ & $7.54 \pm 1.08$ & $8.37 \pm 1.09$ & $11.9 \pm 0.8^{t}$ & $8.39 \pm 1.46$ & $10.8 \pm 0.9^{*}$ \\
\hline $\begin{array}{l}\text { Glc }+ \text { Glyc/(total signal [excluding } \\
\text { residual water] in CMPG sequence) } \times \\
100 \%\end{array}$ & $8.4 \pm 0.6$ & $9.6 \pm 0.6$ & $10.5 \pm 0.7$ & $11.9 \pm 0.7$ & $9.6 \pm 0.5^{*}$ & $13.5 \pm 0.5^{*}$ \\
\hline \multicolumn{7}{|l|}{ Clinical chemistry } \\
\hline TBil $(\mu \mathrm{mol} / 1)$ & $4.6 \pm 0.2$ & $14.9 \pm 2.8^{\dagger}$ & $4.7 \pm 0.4$ & $44 \pm 5^{\star}$ & $3.9 \pm 0.2$ & $133 \pm 15^{*}$ \\
\hline ALP (IU/l) & $216 \pm 7^{\dagger}$ & $316 \pm 13^{\dagger}$ & $261 \pm 17$ & $392 \pm 37 \%$ & $200 \pm 10$ & $374 \pm 17^{*}$ \\
\hline Albumin (g/l) & $13.9 \pm 0.4$ & $12.8 \pm 0.4$ & $15.4 \pm 0.4$ & $11.4 \pm 0.8 *$ & $14.6 \pm 0.5$ & $11.5 \pm 0.7^{*}$ \\
\hline ALT (IU/l) & $55 \pm 7$ & $80 \pm 11$ & $80 \pm 13$ & $69 \pm 10$ & $42 \pm 4$ & $117 \pm 19^{*}$ \\
\hline AST (IU/l) & $233 \pm 39$ & $374 \pm 50$ & $282 \pm 55$ & $277 \pm 51$ & $254 \pm 30$ & $750 \pm 98^{*}$ \\
\hline Chol (mmol/l) & $2.2 \pm 0.2$ & $2.12 \pm 0.08$ & $1.9 \pm 0.1$ & $2.8 \pm 0.3^{t}$ & $2.0 \pm 0.1$ & $2.5 \pm 0.2$ \\
\hline
\end{tabular}


TABLE 2

Incidence and Severity of Microscopic Histological Findings in the Livers of Animals Treated With ANIT and PFCs at 14 Days

\begin{tabular}{|c|c|c|c|c|c|c|}
\hline Group ( $n=8$ per group) & PFC_0.025\% & ANIT_0.025\% & PFC_0.04\% & PFC_0.05\% & PFC_0.05\% & ANIT_0.05\% \\
\hline \multicolumn{7}{|l|}{ Biliary hyperplasia } \\
\hline NAD & 8 & 0 & 8 & 0 & 8 & 0 \\
\hline Minimal & 0 & 0 & 0 & 0 & 0 & 0 \\
\hline Mild & 0 & 4 & 0 & 1 & 0 & 1 \\
\hline Moderate & 0 & 4 & 0 & 7 & 0 & 3 \\
\hline Marked & 0 & 0 & 0 & 0 & 0 & 4 \\
\hline \multicolumn{7}{|l|}{ Peribiliary inflammation } \\
\hline NAD & 4 & 0 & 4 & 0 & 2 & 0 \\
\hline Minimal & 4 & 2 & 4 & 1 & 6 & 0 \\
\hline Mild & 0 & 6 & 0 & 7 & 0 & 8 \\
\hline \multicolumn{7}{|l|}{ Peribiliary fibrosis } \\
\hline NAD & 8 & 0 & 8 & 0 & 8 & 0 \\
\hline Minimal & 0 & 1 & 0 & 0 & 0 & 0 \\
\hline Mild & 0 & 6 & 0 & 8 & 0 & 6 \\
\hline Moderate & 0 & 1 & 0 & 0 & 0 & 2 \\
\hline \multicolumn{7}{|l|}{ Focal hepatocellular necrosis } \\
\hline NAD & 8 & 1 & 8 & 0 & 8 & 0 \\
\hline Minimal & 0 & 4 & 0 & 2 & 0 & 2 \\
\hline Mild & 0 & 3 & 0 & 4 & 0 & 2 \\
\hline Moderate & 0 & 0 & 0 & 2 & 0 & 4 \\
\hline
\end{tabular}

Notes. Eight animals were assessed from each group. Animals are scored for each histological assessment as no abnormalities detected (NAD), minimal, mild, moderate, and marked. Sections were assessed for biliary abnormalities including biliary hyperplasia, peribiliary inflammation, peribiliary fibrosis, and hepatic damage assessments by the detection of focal hepatocellular necrosis. The number of animals exhibiting each abnormalities is given with the severity. 